\title{
Franki Raffles, photographe engagée : la photographie féministe en Écosse dans les années 1980 et 1990
}

Franki Raffles, militant photographer: feminist photography in Scotland in the 1980s and 1990s

\section{Marine Benoit-Blain}

\section{OpenEdition}

\section{Journals}

Édition électronique

URL : http://journals.openedition.org/cel/555

DOI : $10.4000 /$ cel. 555

ISSN : 2262-208X

\section{Éditeur}

École du Louvre

\section{Référence électronique}

Marine Benoit-Blain, « Franki Raffles, photographe engagée : la photographie féministe en Écosse dans les années 1980 et 1990 ", Les Cahiers de l'École du Louvre [En ligne], 10 | 2017, mis en ligne le 03 mai 2017, consulté le 10 décembre 2020. URL : http://journals.openedition.org/cel/555 ; DOI : https:// doi.org/10.4000/cel.555

Ce document a été généré automatiquement le 10 décembre 2020.

\section{c)}

Les Cahiers de l'École du Louvre sont mis à disposition selon les termes de la licence Creative Commons Attribution - Pas d'Utilisation Commerciale - Pas de Modification 4.0 International. 


\title{
Franki Raffles, photographe engagée : la photographie féministe en Écosse dans les années 1980 et 1990
}

\author{
Franki Raffles, militant photographer: feminist photography in Scotland in the \\ 1980s and 1990s
}

Marine Benoit-Blain

1 L'université de St Andrews en Écosse possède l'une des plus importantes collections d'archives photographiques du Royaume-Uni, collection qu'elle doit à sa position d'avant-garde dans l'histoire de la photographie : c'est à partir de St Andrews que David Brewster relaie les découvertes de Fox Talbot en Angleterre, et invente et diffuse la photographie pour la première fois en Écosse ${ }^{1}$. Depuis plus de 150 ans, la collection n'a de cesse de grandir et de s'ouvrir aux pratiques les plus contemporaines. En décembre 2014, la bibliothèque des Collections Spéciales de l'université accueille un fonds resté dormant depuis plus de vingt ans, qui vient l'enrichir d'une diversité nouvelle. Il s'agit du fonds d'archives et d'images de la photographe Franki Raffles, décédée en 1994 après presque quatorze ans d'activité. Avec la complicité d'Alistair Scott, professeur de cinéma à l'université d'Édimbourg et ami d'enfance de la photographe, et de Marc Boulay, alors conservateur de la photographie à la bibliothèque, la collection a pu sortir de l'ombre et faire une arrivée tardive dans les collections, révélant au monde universitaire toute sa richesse, après deux décennies de silence.

2 Qui est Franki Raffles et que nous révèlent ces archives? Photographe engagée et militante, Franki Raffles est profondément ancrée dans une seconde moitié du vingtième siècle marquée par les politiques de la représentation et des relations de pouvoir qui y sont attachées. Au cours d'une carrière brève mais prolifique, s'étendant de 1980 à sa mort en 1994, Franki Raffles se consacre à une pratique passionnée, politisée et volontaire, qui se concentre sur la photographie documentaire des femmes, 
et plus particulièrement des femmes de la classe ouvrière, notamment dans leur cadre de travail. Franki Raffles fait de la photographie un outil au service de sa conviction et de son engagement féministe, auprès de ceux - et celles - qui souffrent le plus des dynamiques de représentation à sens unique.

$\mathrm{Au}$ fil de ses archives et de ses récits, se dessine le portrait sensible d'une femme militante et décidée, qui parcourt le Royaume-Uni, l'Europe et le monde au service de sa cause. À la fois symbolique et profondément humaine, sa trajectoire de photographe se dessine tout en nuances et en passions. Résolument engagée, sa pratique de la photographie entrelace intimement le personnel et le politique et sème les graines d'une construction nouvelle de la photographie.

\section{L'engagement d'une vie : éléments biographiques}

3 Franki Raffles naît en 1955 à Salford, dans la région de Manchester au Royaume-Uni. Sa famille, issue de l'immigration polonaise et enrichie par l'industrie, est à la tête d'une usine d'imperméables à Ancoats, non loin de Salford. Alors que son père est décrit comme un homme d'affaires, sa mère, Gillian Raffles, est galeriste: elle dirige la Mercury Gallery à Londres, où elle expose quelques-uns des plus grands noms des jeunes artistes figuratifs écossais de l'époque, tels qu'Anne Redpath, John Houston ou encore Elizabeth Blackadder, qui deviendra membre de l'Académie Royale de Peinture. Les éléments sont réunis pour que Franki Raffles passe une enfance aisée et cultivée, élève à la prestigieuse école pour filles Lady Eleanor Holles à Hampton dans la banlieue de Londres.

\section{St Andrews et les débuts du militantisme}

On trouve les premières traces d'un engagement militant actif dans la vie de Franki Raffles à partir de 1973. Alors étudiante en philosophie morale à l'université de St Andrews en Écosse, elle s'engage auprès du WLM, le Women's Liberation Movement. Cet engagement politique la mène à ses premiers actes artistiques, lorsqu'elle sérigraphie le logo du WLM sur divers supports. Le contexte particulier de St Andrews n'est pas anodin pour le développement du militantisme de la jeune Franki: l'université de St Andrews est alors reconnue pour ses valeurs conservatrices. En réaction à ce traditionalisme ambiant, les organisations féministes y redoublent d'activité: l'historienne Sarah Browne considère la petite ville comme un "véritable foyer du féminisme ${ }^{2}$ » de l'époque. St Andrews devient ainsi l'une des premières villes en Écosse à voir naître un mouvement de libération des femmes, notamment en raison de la nature essentiellement universitaire de sa population, une dynamique décuplée par l'isolation géographique de sa position côtière. Émerge ainsi un féminisme radical et activiste, au sein duquel Franki Raffles fait ses premières armes en tant que militante. Opposée à la négociation avec les figures d'autorité du patriarcat, elle rédige alors plusieurs articles pour des publications locales, où elle défend un féminisme de la confrontation et de la révolution. Paula Jennings résume leur démarche comme suit : "Le féminisme a comme but de détruire la suprématie masculine et il n'y a rien de calme ou de distingué à ce propos. Être féministe, c'est être en colère ${ }^{3}$ ". Cette approche révolutionnaire du féminisme - frontalement opposée à un féminisme plus pacifié, prôné par certaines branches du WLM à la même époque - est notamment relayée par Franki Raffles en 1978 lorsqu'elle prend la plume dans, au moins, trois publications 
féministes pour réclamer la "rébellion des femmes»: la Tayside Women's Liberation Newsletter ${ }^{4}$, la Brighton and Hove Women's Liberation Newsletter ${ }^{5}$ et WIRES ${ }^{6}$. Dès ses années formatrices, et malgré les critiques et les réserves de son propre mouvement, Franki Raffles se bat pour un féminisme sans compromis : «Aucune femme n'est libérée tant que toutes les femmes ne sont pas libres ", clame-t-elle alors.

\section{Lewis photographiée : premières expérimentations photographiques}

5 À cette même époque, Franki Raffles s'investit en politique et fréquente des cercles communistes et marxistes, sans pour autant jamais rejoindre le parti. Son intérêt politique prépondérant pour la cause féministe se lie donc à une forte préoccupation politique communiste d'orientation marxiste-léniniste. Elle quitte St Andrews à la fin de ses études en 1977 : mue par un désir de vie en autarcie et en communauté, fruit de son époque, elle s'installe alors sur l'île de Lewis, dans les Hébrides extérieures du nordouest de l'Écosse. Elle y achète l'ancien bureau de poste du village, dans un état proche de la ruine. Lieu aride et difficile, très peu peuplé, Lewis avait déjà fasciné le photographe Paul Strand, qui, en 1954, avait vu dans la vie en communauté des habitants de Lewis une sorte d'idéal utopique communiste ${ }^{7}$. C'est à Lewis que Franki Raffles installe sa chambre noire et développe ses premières images. Autodidacte, elle y perfectionne sa pratique photographique, se concentrant sur le noir et blanc - et, déjà, sur les femmes de l'île, qu'elle photographie dans leurs activités quotidiennes. En 1980, elle réalise sa première exposition, sobrement intitulée Lewis Women. Sa première photographie est publiée en 1981 : elle illustre le mois d'octobre de l'année 1982 dans le calendrier féministe Her Stories - publié par les éditions féministes Stramullion - avec le portrait d'une femme de l'île. La critique est élogieuse : la journaliste Ann Shaw la mentionne dans le quotidien Glasgow Herald comme "l'une des images les plus marquantes d'un point de vue photographique ${ }^{8} »$.

\section{We Can Take Pictures : photographie participative et éthique de la communication}

$6 \quad$ Franki Raffles quitte Lewis après y avoir passé quatre ans et rejoint Édimbourg pour y pratiquer la photographie de manière professionnelle. Dès le début, elle s'engage dans une pratique de la photographie éthique et sociale, qui se concentre plus sur le sujet que l'objet photographique. Le projet We Can Take Pictures, réalisé entre 1982 et 1983, témoigne tout particulièrement de cet engagement social en son sens le plus large, ainsi que de son rôle précurseur dans ce champ. En collaboration avec une école de la ville, Franki Raffles organise des ateliers de photographie où elle apprend à des enfants en situation de handicap à manipuler des appareils Polaroid. Elle propose ensuite une mise en abyme du projet en photographiant les enfants pratiquant la photographie, exprimant l'émotion de leur premier contact avec le médium et l'interaction immédiate permise par l'utilisation du processus Polaroid. Doigts pointés, jeux de regard, étonnement et joie de vivre traduisent dans ces images le dialogue que la photographie permet d'établir pour des enfants en mal de parole (fig. 1). Ce qui est en jeu ici est bien la construction d'un discours sur le pouvoir de communication de la photographie, qui devient un moyen d'expression et de parole pour des voix en difficulté. Cette pratique de la photographie participative ne sera véritablement développée dans les milieux scolaires qu'à partir des années 1990 avec un afflux d'apports théoriques et 
sociologiques', soit près d'une décennie après l'action de Franki Raffles, qui préfigure ainsi une pratique pédagogique de grande ampleur ${ }^{10}$.

Fig. 1

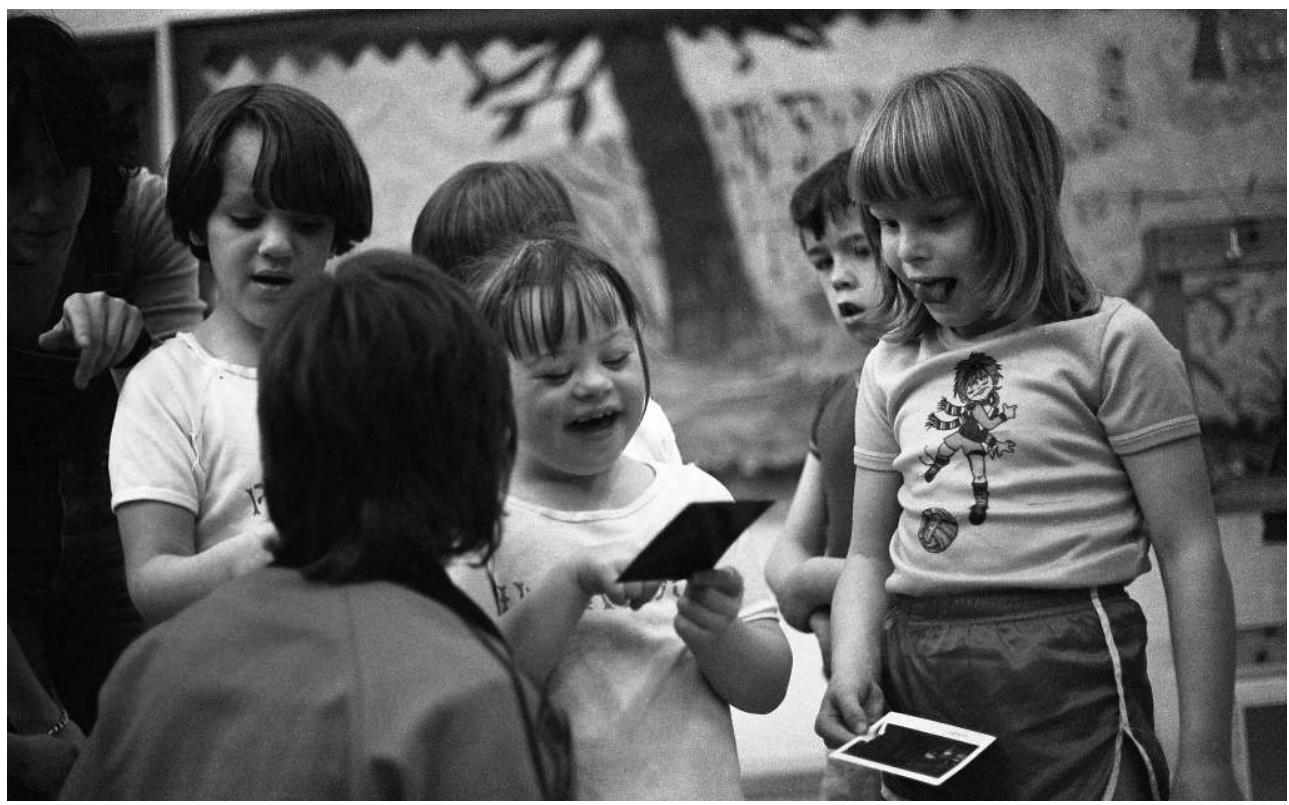

Franki Raffles, Projet We Can Take Pictures, Édimbourg, 1982, Franki Raffles Photography Collection (1979-1994) 2014-4-T-TP025

(c) Bibliothèque des collections spéciales de l'université de St Andrews, Royaume-Uni

7 Dès lors, les projets s'enchaînent, toujours marqués par cette volonté d'illustrer la vie des femmes, avec une sensibilité picturale en développement constant. Elle refuse de pratiquer la photographie commerciale et le choix de ses commanditaires témoigne d'un investissement social et politique toujours grandissant: on y trouve entre autres le Conseil des Femmes d'Édimbourg, le Conseil régional de Strathclyde, qui œuvre à l'égalité des femmes dans le travail, ou encore la chaîne télévisée BBC que Franki Raffles accompagne lors de la réalisation du documentaire Putting Women in the Picture en 1987.

8 Au-delà de ces commandes locales, Franki Raffles se révèle aussi une grande voyageuse : entre 1984 et 1985, elle arpente l'Asie; puis, en qualité de photographe professionnelle, le Mexique en 1986, l'URSS en 1989 et Israël en 1993. Ces périples qui jalonnent sa carrière déterminent l'axe directeur de sa pratique photographique et de son engagement personnel : elle photographie la condition féminine de par le monde, avec une éthique internationaliste issue de son militantisme communiste, qui la pousse à s'intéresser à la condition de toutes les femmes de la classe ouvrière.

\section{Femmes au travail : substance politique et stratégies documentaires}

\section{Le travail, symbole d'indépendance}

9 Sa fascination pour la représentation des femmes de la classe ouvrière se traduit par un engagement à leurs côtés. Dans ses images, un élément clé se distingue : le travail, 
symbole de l'accès des femmes à l'indépendance et à l'autonomie. Cet engagement est particulièrement marquant dans les photographies prises en URSS pendant l'été 1989 : accompagnée de sa fille Anna, alors âgée de dix ans, Franki Raffles documente de manière ambitieuse de nombreux exemples de lieux où sont employées des femmes: son périple soviétique devient alors la tentative d'un inventaire exhaustif du travail féminin dans un pays qui prône l'égalité salariale. Usines de bois, de viande, fermes, hôpitaux, écoles : à travers des déclinaisons du travail, on retrouve des femmes actives, efficaces, fortes. Sous l'appareil de Franki Raffles, les femmes réalisent toutes les tâches que l'on avait pu voir les hommes réaliser dans le passé, évoquant subtilement les images de propagande soviétique montrant des figures masculines redoublant de productivité. Les femmes sont ici montrées au cœur des machines, qu'elles manient avec compétence et confiance : dans des images presque manichéennes, les ouvrières, au cœur de constructions géométriques industrielles, sont baignées dans un halo de lumière qui vient révéler leur travail, sérieux et précis (fig. 2). Les figures féminines ainsi présentées viennent remplacer les héros masculins de l'imaginaire populaire.

Fig. 2

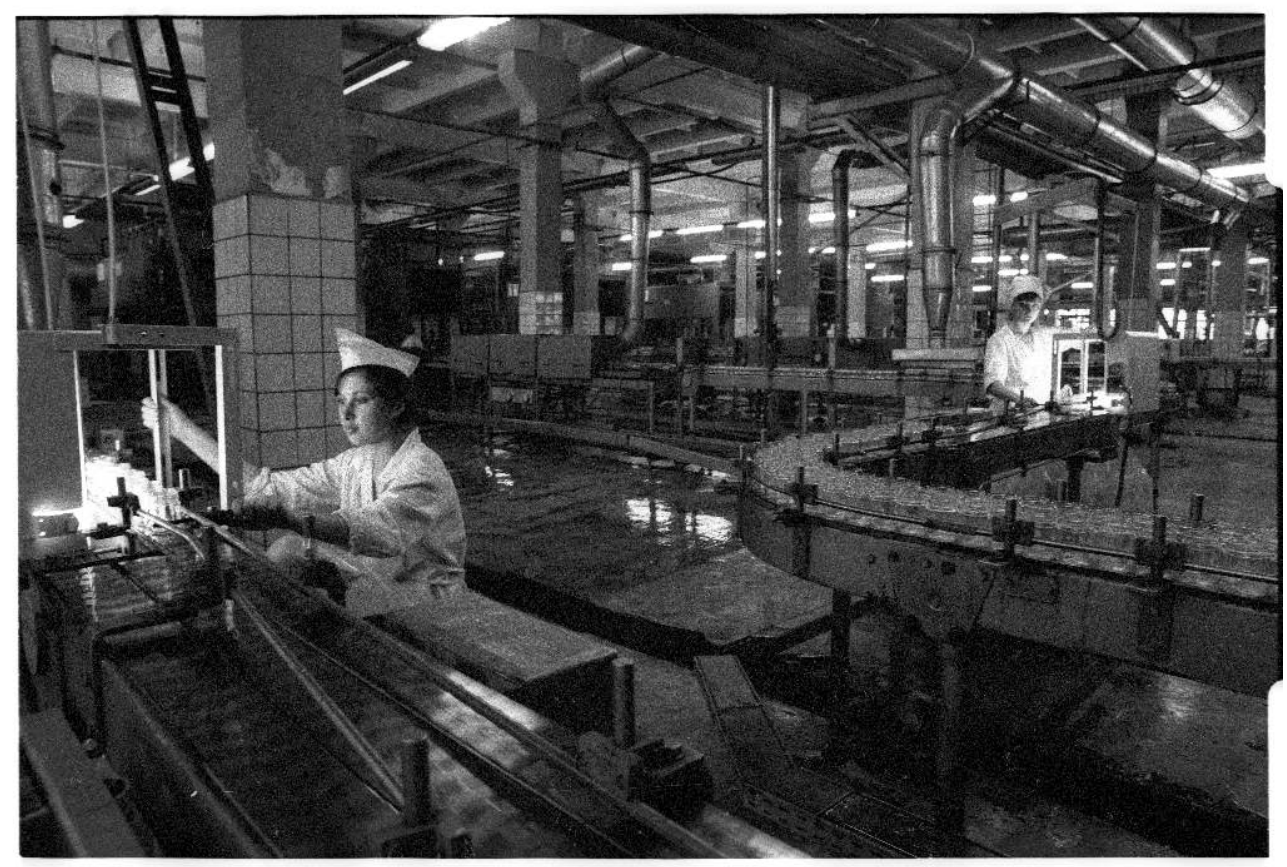

Franki Raffles, Projet Women Workers, URSS, 1989, Franki Raffles Photography Collection (1979-1994) 2014-4-038-5a

(c) Bibliothèque des collections spéciales de l'université de St Andrews, Royaume-Uni

\section{Les femmes au cœur du tableau}

Ces images de la femme s'ancrent ainsi dans une dimension résolument active du sujet, en référence explicite et militante à la tradition de la représentation de la femme comme objet passif de la représentation. Soulevée par Linda Nochlin en 1970 dans son célèbre article « Pourquoi n'y a-t-il pas eu de grandes femmes artistes ${ }^{11}$ », la question de la représentation des femmes dans l'histoire de l'art découle de la relation prépondérante entre celui qui voit et celui qui est vu, le voyeur et sa victime. Là où de 
nombreuses artistes des années 1960 et 1970 cherchent à renverser cette dynamique en se réappropriant la question du corps par un travail performatif, Franki Raffles s'applique ici à remettre au cœur du tableau des femmes qui sont sujets sans être objets. Ainsi, les figures féminines sont toutes conscientes d'être photographiées, et choisissent leur façon d'être représentées. En outre, les visages souriants, témoignant d'une claire joie de vivre, échappent au statut de victime souvent conféré aux sujets de la photographie documentaire sociale dans son sens le plus classique. Les femmes photographiées par Franki Raffles font preuve, à travers leur travail, de leur capacité à exister entièrement en dehors de la sphère masculine. Les hommes sont d'ailleurs les grands absents de l'inventaire sociologique de la photographe : leurs rares incursions les trouvent en position passive, apparaissant par exemple comme les patients d'une docteur soviétique. Assis, en périphérie de l'image, silencieux, le dos tourné, les hommes sont évincés du paysage social que dessine Franki Raffles : la parole est donnée aux femmes, au nom de plusieurs siècles de silence.

\section{Célébrer la communauté féminine}

11 La sphère féminine est donc montrée comme autosuffisante et indépendante. En outre, elle est profondément fondée sur une solidarité interféminine, une fraternité - peutêtre devrait-on parler de sororité - qui se construit sur une expérience féminine propre à la communauté formée par les sujets féminins. Les relations interféminines et la mise en valeur d'un véritable réseau féminin deviennent un des thèmes les plus récurrents chez Franki Raffles : qu'elle soit en Chine ou à Édimbourg, elle ne photographie pas tant les femmes qu'elle ne photographie les femmes ensemble. Ce qui est vrai pour le travail à la chaîne des femmes dans les usines d'Édimbourg l'est aussi pour les vues plus intimes des femmes israéliennes photographiées chez elles: la multiplicité des contextes et des histoires ne fait que mieux ressortir ce leitmotiv sensible qui voit émerger une communauté féminine. L'une des occurrences les plus évidentes de ce thème se trouve peut-être lors des Commonwealth Games d'Édimbourg en 1986 : Franki Raffles y photographie les athlètes féminines tout comme les spectatrices. Les femmes y sont photographiées ensemble, fêtant leurs victoires, se soutenant les unes les autres, à l'image de cette photographie de deux athlètes se serrant la main en passant la ligne d'arrivée (fig. 3). La photographie devient ici un outil de célébration des femmes dans leur existence la plus multiple et la plus capable. 
Fig. 3

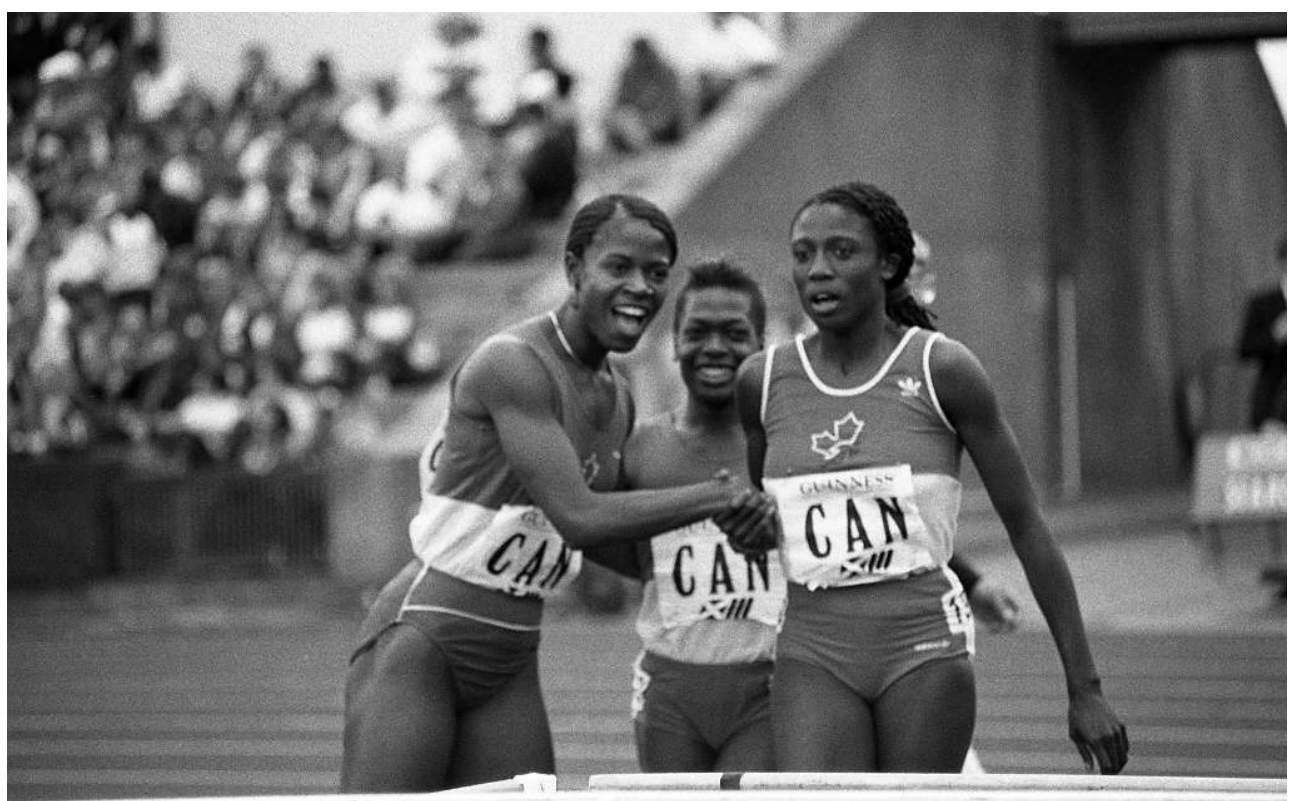

Franki Raffles, Projet Simply Women, Édimbourg, 1986, Franki Raffles Photography Collection (1979-1994) 2014-4-T-SP005

(C) Bibliothèque des collections spéciales de l'université de St Andrews, Royaume-Uni

\section{La photographie documentaire en question}

Il y a chez Franki Raffles une véritable exaltation du sujet, qui s'oppose à une tradition d'aliénation: une très forte conscience du poids politique de la photographie, et plus particulièrement de la photographie documentaire. Il s'agit de prendre le contre-pied d'une critique notamment formulée par Martha Rosler, qui estime que le genre documentaire "prend le rôle d'un film d'horreur, mettant un visage sur nos peurs et transformant la menace en fantasme, en imagerie ", que l'on "peut supporter en le laissant derrière nous: c'est eux, pas nous ". Martha Rosler décrit ainsi un "documentaire libéral qui calme toute agitation de conscience chez ses spectateurs de la même manière que gratter calme une piqûre, et les rassure en même temps sur leur richesse relative et leur position sociale ${ }^{12} »$. À cette conception du documentaire "libéral», peut-être faudrait-il opposer une pratique d'un documentaire "communiste", ou au moins "commun", dans le sens de commun à tous. Les photographies de Franki Raffles évoquent une relation directe entre la représentation et le don de la parole. Dans un débat féministe qui met alors en avant l'invisibilité de la place de la femme dans la société, la photographe comprend que les outils de représentation sont aussi et surtout des outils de revendication politique.

Ainsi, loin du sensationnalisme de la misère évoqué par Martha Rosler, la pratique photographique de Franki Raffles se distingue aussi par un intérêt pour les temps invisibles. Dépassant une vision productiviste du travail, la photographe cherche à mettre en lumière l'existence d'un travail invisible, qui existe en dehors des paramètres étroits de la représentation habituelle du travail, et qui met en valeur l'apport de femmes notamment dans l'industrie du service. C'est aussi ce qui guide son intérêt pour le monde de l'éducation, non seulement avec We Can Take Pictures, tel que cité plus en amont, mais aussi à travers le projet Picturing Women qui prend place entre 1988 
et 1989. La photographe passe alors près d'un an dans une école d'Édimbourg, où elle ne photographie cette fois-ci pas les enfants mais les employées : institutrices, directrices, femmes de ménage, cuisinières. Ce projet est tout d'abord l'occasion de révéler les travailleuses de l'invisible, celles qui n'ont ni produit fini ni reconnaissance, à l'instar d'une image montrant l'institutrice seule dans la classe vide après la fin de la journée, préparant sa journée à venir (fig. 4), ou trois cuisinières discutant en cuisine. En outre, ce projet évoque un élément plus complexe encore, en écho avec les revendications politiques de la photographe : il s'agit des relations de hiérarchie que reproduisent les femmes entre elles. Son investigation photographique dans les murs de l'école révèle les constructions hiérarchiques dénoncées dans les rapports de force du patriarcat, reproduits ici entre les différentes actrices de la vie de l'école. La photographie de l'invisible est donc double, entre célébration du travail ignoré de ces femmes et dénonciation des rapports toxiques qui y sont reproduits. Une photographie rend particulièrement tangible ce rapport de force implicite: on y voit une femme de ménage courbée et de dos, balai à la main, discutant avec la directrice de l'école alors que celle-ci se tient en haut des escaliers, de face.

Fig. 4

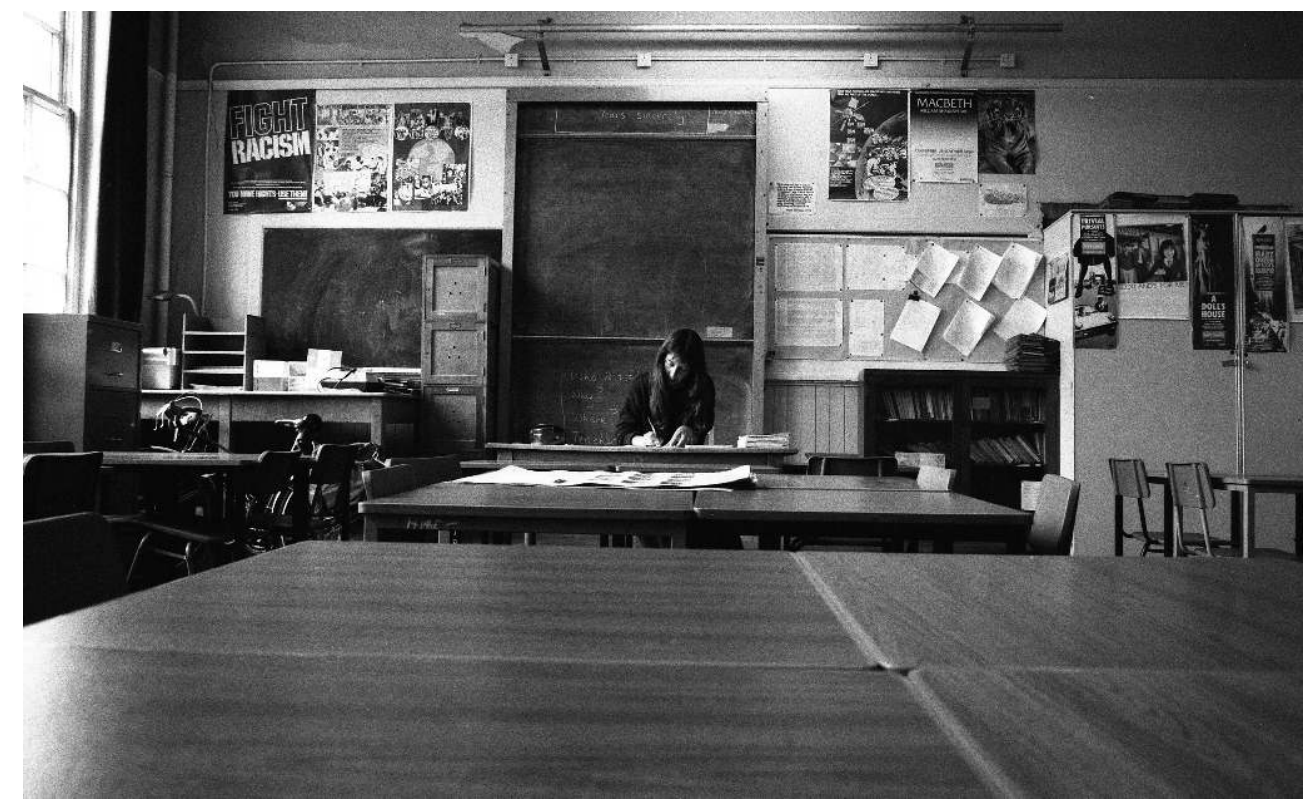

Franki Raffles, Projet Picturing Women, Édimbourg, 1988, Franki Raffles Photography Collection (1979-1994) 2014-4-T-PW013

(C) Bibliothèque des collections spéciales de l'université de St Andrews, Royaume-Uni

\section{La postérité en question : quel avenir pour les pratiques hybrides et politiques?}

14 Pourquoi une œuvre aussi multiple, foisonnante et passionnée a-t-elle mis plus de vingt ans à redevenir visible? Le projet Zero Tolerance de Franki Raffles permet de questionner la façon dont l'hybridité d'une production définit son mode de diffusion et détermine son accès à la postérité, tout en ouvrant de nouveaux lieux d'investigation pour la recherche contemporaine. 


\section{Zero Tolerance, l'image au service du discours}

15

$$
\begin{aligned}
& \text { 1994, et peut-être aussi le plus ambitieux dans son ampleur, et le plus important dans } \\
& \text { sa réception. Campagne consacrée à la dénonciation des violences faites aux femmes, } \\
& \text { Zero Tolerance devient rapidement à la fois le fer de lance de la carrière de Franki Raffles } \\
& \text { et l'aboutissement de près de quinze ans de pratique militante. Ce projet marque son } \\
& \text { entrée dans la postérité et dans l'histoire de l'Écosse, et lui doit une entrée } \\
& \text { biographique dans plusieurs encyclopédies, dont le Dictionnaire biographique des femmes } \\
& \text { écossaises, où l'on peut lire: } \\
& \text { RAFFLES, Frances Rachel (Franki), née à Salford } 17 \text { oct. 1955, morte à Édimbourg } \\
& 6 \text { déc. 1994. Photographe et cofondatrice de la campagne Zero Tolerance }{ }^{13} \text {. }
\end{aligned}
$$

Zero Tolerance est l'un des derniers projets réalisés par Franki Raffles avant sa mort en

Ce projet commence par une large enquête sociologique sous la forme d'une étude approfondie des violences conjugales en Écosse. Le sujet est alors tabou, et Franki Raffles, en collaboration avec Evelyn Gillan du Conseil de la ville d'Édimbourg, entreprend de le rendre visible et de dénoncer de façon systématique les préjugés et les mensonges qui l'entourent. Commence alors une large campagne construite autour de la lettre $\mathrm{Z}$, qui devient un véritable logo pour les militants impliqués et marque fortement l'identité visuelle des rues d'Édimbourg pendant la durée de la campagne. La stratégie photographique adoptée est celle d'une relation forte entre image et texte, fondée sur des photographies montrant des femmes de tout âge vaquant à leurs occupations quotidiennes, et jouant sur le choc créé par les informations textuelles qui révèlent une violence cachée. Jouant à nouveau sur les notions de visible et d'invisible dans l'image, Franki Raffles cherche à montrer la banalité que peuvent prendre les actes de violence domestique, qui ne se lisent pas toujours sur le visage des victimes. On peut ainsi voir une photographie montrant plusieurs adolescentes assises sur un lit, d'apparence insouciante. La légende, pourtant, frappe le spectateur : « Elles ont le droit de dire non. Certains hommes n'écoutent pas. » (fig. 5). 
Fig. 5

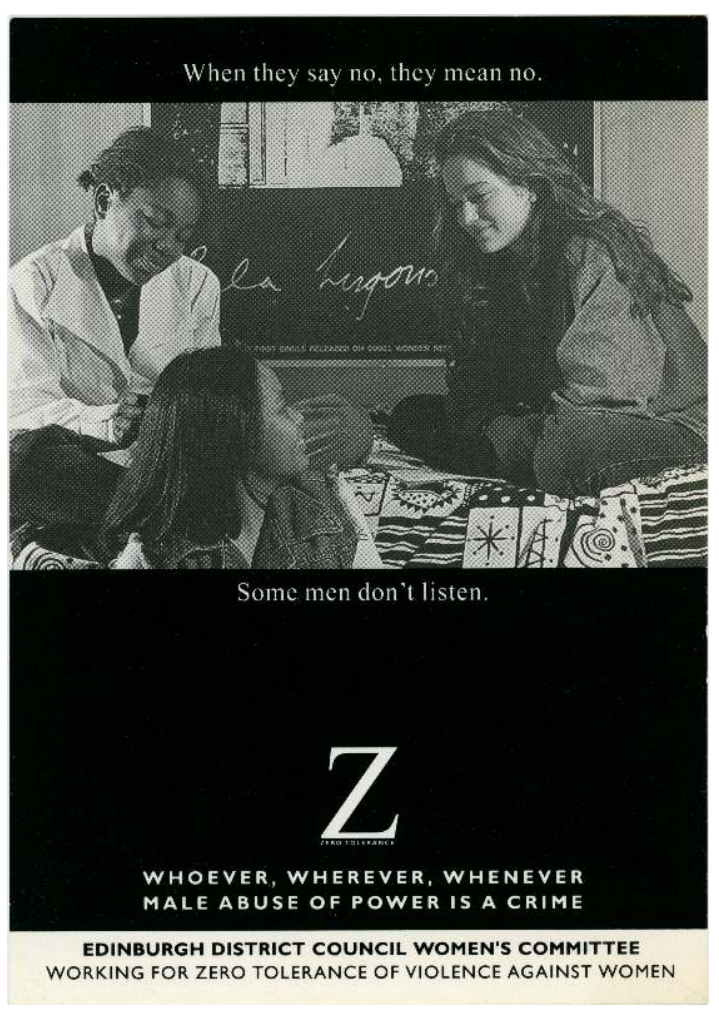

FRANKI RAFFLES, PROJET ZERo TOLERANCE, ÉdIMBOURg, 1991-1992, CARTE DE PRÉSENTATION, IMPRESSION TYPOGRAPHIQUE ET SIMILIGRAVURE, FRANKI RAFFLES PHOTOgRAPHY COLLECTION (1979-1994) 2014-4-ZT061

(C) Bibliothèque des collections spéciales de l'université de St Andrews, Royaume-Uni

Pour la photographe, il est nécessaire de comprendre l'aspect quotidien pris par les violences domestiques, qui tient d'un statu quo qu'elle décrit en ces termes:

Il est important lorsque l'on parle de la question du viol, que l'on ne le perçoive pas comme un crime sexuel. Cela fait partie d'un spectre de crimes de violence contre les femmes, perpétués par les hommes [...]. Les crimes de violence contre les femmes sont des abus de pouvoir. Les hommes continueront d'abuser de ce pouvoir tant que l'on n'aura pas un climat d'opinion qui rend ces abus inacceptables ${ }^{14}$.

Si son but est donc de détruire un état de fait qui rend non seulement les violences contre les femmes possibles, mais surtout silencieuses et acceptées, le résultat est à la hauteur de ses espérances. La campagne, diffusée à grande échelle dans la ville d'Édimbourg, puis de Glasgow, et enfin étendue à plusieurs villes du Royaume-Uni, provoque un raz-de-marée dans le paysage politique et culturel écossais de l'époque. En réponse à une campagne absolument inédite qui inonde les rues de la ville, forte de sa puissance visuelle et attaquant frontalement le tabou, les réactions sont vives. Un journaliste du Sunday Times parle de la «disgrâce dans une société démocratique mature » de ce qu'il compare à un « exercice de propagande par la haine à la Goebbels ${ }^{15}$ ». "Ces affiches peuvent faire que de nombreux enfants ne vont plus se sentir en sécurité avec leurs pères ou leurs oncles. C'est très certainement le message de ces affiches et je ne suis pas d'accord ${ }^{16}$ ", peut-on encore lire. La mise en évidence d'un lien entre masculinité, violence et crimes sexuels, forçant le spectateur à affronter la réalité des violences domestiques de manière aussi irréfutable que visuellement percutante, 
crée une véritable onde de choc dans une ville encore très conservatrice. Lesley Riddoch résume le phénomène en une phrase :

On n'avait jamais pris un message pareil, un message aussi controversé, pour le faire passer par des médias aussi puissants, globaux, conventionnels ${ }^{17}$.

\section{Postérité politique et postérité artistique}

La soudaine mort en couches de Franki Raffles en 1994 met fin à sa carrière ascendante de photographe et de militante, mais ne met pas fin à la campagne Zero Tolerance qui continue de prendre de l'ampleur, jusqu'à la création d'une branche new-yorkaise menée par Jennifer McCarey en 1995. Pour autant, malgré l'indéniable postérité politique du projet Zero Tolerance, les photographies restent dans l'ombre après sa mort, dans un placard de la demeure familiale qu'occupent sa compagne Sandy Lunan et leurs enfants. Si l'héritage politique est fort, il éclipse le reste des contributions de Franki Raffles à l'histoire de la photographie, jusqu'à l'intervention d'Alistair Scott et l'arrivée des œuvres dans une infrastructure de conservation perfectionnée pour l'accueil des archives photographiques à St Andrews.

19 L'histoire d'un archivage si difficile, mais aussi et surtout d'une diffusion si tardive, est celle de tout un pan de la production photographique de cette époque. Zero Tolerance, entre image et texte, nous montre que Franki Raffles pratique une photographie tout à fait hybride, à mi-chemin entre production d'images et activisme politique en son sens le plus littéral. Définie par sa destination, l'image photographique se fait l'outil du message dialectique. Du fait d'une pratique avant tout définie par son auteure comme une pratique militante, aucune de ses images n'a de réelle présence sur le marché de l'art. Ceci est irréfutablement lié à l'engagement de Franki Raffles, qui souhaitait diffuser et montrer ses images au plus près de ceux qu'elle photographiait - et non dans des galeries, qu'elle ne fréquentait qu'occasionnellement. Ainsi, nombre de ses photographies sont exposées dans des centres sportifs, des bibliothèques, ou d'autres lieux adoptés par la communauté ouvrière ${ }^{18}$. Un deuxième leitmotiv vient définir la diffusion de ces images: les lieux de diffusion féministes ont souvent une présence alternative, éloignée des réseaux traditionnels du marché de l'art. On peut citer à ce titre le First of May Bookshop : espace politiquement positionné à gauche et participant à la publication d'ouvrages dédiés à la communauté homosexuelle, au féminisme et au socialisme en Europe, hébergeant les éditions Stramullion ${ }^{19}$, il est cité par le journal Marxism Today comme l'une des « librairies radicales de Grande-Bretagne ${ }^{20} »$. Cet espace accueille à plusieurs reprises des expositions de tirages photographiques de Franki Raffles, et notamment ses photos de la Chine. Loin du réseau muséal et des lieux délimités de l'art contemporain de l'époque, ces espaces sont tout autant de ressources aujourd'hui pour les chercheurs intéressés par la production de ces cultures alternatives.

\section{Conclusion}

20 La carrière de Franki Raffles est celle d'une photographe à l'avant-garde, audacieuse, profondément militante et sans compromis: ouverte sur le monde, passionnée et sensible, elle a consacré sa vie à la subversion d'une perception passive et classique de la femme. 
Au-delà d'une seule dénonciation des mécanismes d'oppression qui définissent les constructions sociales du genre, Franki Raffles s'inscrit dans la lignée de Linda Nochlin qui déclarait que «les politiques féministes d'aujourd'hui sont beaucoup plus polyvalentes et conscientes d'elles-mêmes : les lignes de bataille sont moins clairement délimitées ${ }^{21} »$. C'est bien la conscience d'elle-même et la conscience de sa pratique qui délimitent la démarche de Franki Raffles. À travers son œuvre, la photographe recherche un nouveau type de production de discours, visant à la révolution systématique des modes de représentation. Elle s'empare ainsi de l'appareil photographique en étant pleinement consciente de ses capacités et de ses limites : elle fait de la production d'images, que Carol Armstrong avait pu comparer à « un acte prédateur de violation, comme une forme de possession symbolique ${ }^{22} »$, un instrument de communication et d'expression pour des figures marginalisées de l'histoire de la représentation. De l'usage de la photographie participative à l'introduction de l'écrit comme système dans lequel le sujet peut collaborer avec le créateur d'images, Franki Raffles développe une éthique du sujet qui la pousse à questionner la fabrique même de la photographie.

21 Cette vision est celle d'une photographie qui est donc avant tout un outil ; il n'existe pas ici de photographie sans engagement militant. Tout comme le militantisme précède la photographie, le message précède l'image et la destination précède l'esthétique. Ainsi, l'engagement féministe est premier dans sa carrière, et de là naît l'exercice de la photographie, et non l'inverse.

Cet engagement au plus près du sujet, qui s'applique aussi à une pratique de la diffusion de son œuvre, explique que son existence dans les milieux alternatifs et engagés de l'Écosse des années 1980 et 1990 a privé son œuvre de la postérité jusqu'à ce jour. La dynamique de cette typologie de production est donc à double tranchant: reconnue pour sa dimension politique et militante, elle jouit d'une véritable existence dans le champ de l'histoire politique ; tandis qu'elle tend, parallèlement, à échapper à l'œil de l'histoire de l'art et de l'histoire de la photographie.

\section{NOTES}

1. Tom Normand, Scottish Photography: A History, Édimbourg, Luath Press, 2007, p. 26.

2. Sarah Browne, "A veritable hotbed of feminism": Women's Liberation Movement in St Andrews, c. 1968-c. 1969 », Twentieth Century British History, vol. 23, n 1, 2011, pp. 100-123.

3. Paula Jennings, "The Value of Angry Confrontation in Feminist Strategy ", Catcall: A Feminist Discussion Paper, $\mathrm{n}^{\circ}$ 5, mars 1977, p. 6.

4. Franki Raffles et Paula Jennings, Tayside Women's Liberation Newsletter, juin-juillet 1978, p. 4.

5. Franki Raffles et Paula Jennings, Brighton and Hove Women's Liberation Newsletter, juillet 1978, p. 2.

6. Franki Raffles et Paula Jennings, WIRES, $n^{\circ}$ 52, 1978, non paginé.

7. Paul Strand et Basil Davidson, Tir a'Mhurain, Londres, MacGibbon and Kee, 1962.

8. Ann Shaw, «The Alternative Calendar », The Glasgow Herald, 7 décembre 1981, p. 8. 
9. Johanna Einarsdottir, «Playschool in pictures: children's photographs as a research method ", Early Child Development and Care, vol. 175, n 6, août 2005, p. 525.

10. Idem, Ibidem, p. 527.

11. Linda Nochlin, «Why Have There Been No Great Women Artists? », ARTnews, janvier 1971, p. 22.

12. Martha Rosler, «In, around and afterthoughts (on documentary photography) ", dans Liz Wells (éd.), The Photography Reader, New York, Routledge, 2003, p. 263.

13. Elizabeth Ewan, Sue Innes, Sian Reynolds et Rose Pipes, The Biographical Dictionary of Scottish Women, Oxford, Oxford University Press, 2006, p. 296.

14. Franki's notebook, cahier jaune, Archives Franki Raffles, bibliothèque des Collections Spéciales de l'université de St Andrews.

15. Gerard Warner, "Time to give Zero Tolerance to the sex warriors ", Sunday Times, 9 octobre 1994, p. 8.

16. Norman Irons cité par Rupert Steiner et Neville Rigby, «Zero Tolerance doesn't add up », Sunday Times, 23 octobre 1994, p. 4.

17. Lesley Riddoch dans un entretien avec Alistair Scott réalisé le 27 juin 2013.

18. Catherine Lockerbie, "Franki's focus on women at work », The Glasgow Herald, 12 juillet 1988, p. 4.

19. Gail Chester et Sigrid Nielsen (éd.), In other words: Writing as a feminist, Londres, Routledge,

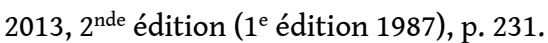

20. Marxism Today, juin 1985, p. 44.

21. Linda Nochlin, telle que citée par Kathy Battista, Renegotiating the Body: Feminist Art in 1970's London, Londres, I.B. Thauris, 2013, p. 7.

22. Carol Armstrong, "The Reflexive and the Possessive View ", Representation, $\mathrm{n}^{\circ} 25$, hiver 1989 , pp. 58-59.

\section{RÉSUMÉS}

Conservé à la bibliothèque des Collections Spéciales de l'université de St Andrews, le fonds d'images et d'archives de la photographe Franki Raffles est le fruit d'une carrière brève mais prolifique, marquée par un activisme politique revendiqué. Franki Raffles (1955-1994) consacre sa vie à la déconstruction des relations de pouvoir inhérentes aux dynamiques de la représentation, notamment dans la photographie documentaire. Son œuvre photographique est ainsi exclusivement dédiée à la représentation des femmes comme figures indépendantes. Fortement influencée par les courants de pensées d'extrême-gauche, et notamment la philosophie marxisteléniniste, elle subvertit une tradition univoque de la représentation pour faire de la photographie un outil de dialogue et de communication. Personnage passionné, complexe et engagé, Franki Raffles devient l'ambassadrice d'une pratique de la photographie comme outil de lutte et de revendication, dans une création militante et féministe.

Kept in the Special Collections Division of the University of St Andrews, the collection of the images and archives of the photographer Franki Raffles (1955-1994) is the fruit of a short but prolific career, marked by political activism. Raffles devoted her life to the deconstruction of the power relations inherent in the dynamics of representation, particularly in documentary photography. Her photographic oeuvre was thus exclusively dedicated to the representation of 
women as independent figures. Heavily influenced by far-left schools of thought, particularly Marxist-Leninist philosophy, she subverted a univocal tradition of representation to make photography a tool for dialogue and communication. A passionate, complex and politically committed figure, Raffles became the ambassador for the practice of photography as a tool for struggle and protest, and her work was militant and feminist.

\section{INDEX}

Mots-clés : photographie documentaire, photographie féministe, féminisme, photographie militante, Écosse, Royaume-Uni

Keywords : documentary photography, feminist photography, feminism, militant photography, Scotland, United Kingdom

\section{AUTEUR}

\section{MARINE BENOIT-BLAIN}

Diplômée d'un master de recherche en histoire des collections de l'École du Louvre, Marine Benoit-Blain se spécialise en histoire de la photographie et étudie notamment l'histoire de la photographie documentaire. À l'occasion d'un séjour de recherche à l'université de St Andrews au Royaume-Uni, elle réalise un mémoire dirigé par Dominique de Font-Réaulx et Michel Poivert dédié à l'étude de la photographie documentaire féministe en Écosse, à travers l'œuvre de Franki Raffles. Elle s'est récemment consacrée à une étude sur les relations entre politiques culturelles et cultures urbaines et participe au plan d'accompagnement de l'art urbain du ministère de la Culture et de la Communication.

$* * *$

The holder of a research degree in the history of collections from the École du Louvre, Marine Benoit-Blain specialises in the history of photography and the history of documentary photography in particular. While conducting research at the University of St Andrews in the United Kingdom, she wrote a thesis, supervised by Dominique de Font-Réaulx and Michel Poivert, dedicated to the study of feminist documentary photography in Scotland, focussing on the work of Franki Raffles. She recently completed a study on the relationship between cultural policies and urban culture, and contributes to the French ministry of culture's support plan for urban art. 